\title{
信息通信工程中传输技术的应用研究
}

\author{
潘燕儿 \\ 浙江省通信产业服务有限公司金华市分公司 \\ DOI:10.32629/btr.v2i12.2695
}

[摘 要] 在科技时代背景下,信息技术逐步拓展应用到通信工程中,并取得了良好的成效。通信工程的重点是通信信号的传输, 为此,将传输技术 应用到信息通信工程中具有重要意义。为此,本文简要介绍了国内传输技术的发展现状,以及传输技术在信息通信工程中的应用情况,预测了传 输技术的未来发展趋势, 以供借鉴。

[关键词] 信息通信工程; 传输技术; 应用情况

全面优化信息通信工程, 能够满足用户的基本需求。同时, 将传输技术 应用到信息通信工程中, 可提升通信信号传输时效性与稳定性, 推动通信 行业的良好发展。为此, 全面探究传输技术在信息通信工程中的实践应用 至关重要。

\section{1 国内传输技术发展现状}

目前, 国内市场的传输产品逐步趋向体量微型化与功能多样化。以智 能手机为例, 其不仅是交互信息和接打电话的工具, 还可以购物、社交、听 音乐、看电影、接发邮件, 这也充分体现出传输技术的功能多样化特征。

传输技术的核心原理是对独立化的设备进行功能集中化设置, 实现设 备功能的时效性联合应用, 增强传输技术的与稳定性。同时, 我国传输产品 的一体化特征也较为突出。通信设备功能的多样化使得传输产品的一体化 程度不断加深, 这也意味着传输产品市场交易价格的提升, 乃至整个传输 产品市场的良好发展。传输产品一体化能够促使传输技术监管人员更加高 效的控制传输技术, 动态化实时控制传输设备, 而这对于推动传输技术产 业的良好发展具有实际意义。

\section{2 传输技术在信息通信工程中的实践应用}

2. 1 传输技术的应用类型与要点

2.1.1 ASON传输系统的特点

信息通信工程中的传输技术, 主要是以形成不同类型的传输系统来实 现的, 同时, 各类传输系统都具有极为鲜明的特点。在实践应用过程中, 可 以结合信息通信工程的基本特征, 配置对应的传输系统, 改进通信质量。在 信息通信工程中, 传输技术的应用可以形成稳定的ASON传输系统。ASON传

行漏电保护器的合理化选择。我们需要依据低压配电系统中电流大小、漏 电保护有关规范来进行漏电保护器的专业化选择, 避免错误保护问题的出 现。第三, 需要对于应用的线路进行科学化布局, 并且需要对于外部环境对 于线路安全性应用的影响进行充分考虑, 充分保障线路的安全和质量, 提 升线路应用的效率。

3. 3 民用高层房建低压配电中的电能设计要点

低压配电的电能设计需要依据《建筑设计防火规范》和民用高层房建 电力系统应用的等级来进行设计工作, 应用较低的成本达到最大的效益 和价值。具体来讲, 第一, 需要依据负荷等级进行电能的科学计算。比如: 依据单位容量法和负荷密度法进行分区的负荷密度计算、然后进行民用 高层房建整体的负荷密度值推算, 并且需要对于无功功率补偿、电能消 耗量进行充分考虑, 提高计算的准确性。第二, 在设计工作中需要对于电 能进行合理化分配, 充分提高电力资源应用的质量和水平。比如：实行 一家一表的方式, 在负一层中安装必要的计量集中箱、然后应用必要电 缆将各家各户住宅配电箱引入到集中类型的配电箱中, 充分的保障设计
输系统以网络智能化为核心实现网络交互链接。ASON传输系统集合了 IP 技术的优势特征, 在实际运行中体现出IP的快捷化特征、SDH的保护功能 特征与WDM的大容量特征, 可以高效整合网络信息资源。ASON传输系统可 以直接提供智能分布式恢复算法和智能光网络路由器, 提高信息通信工 程质量。

2.1.2 MSTP系统和WDM系统的特点

当传输技术在信息通信工程中的应用以MSTP系统来体现时, SDH系统 属于核心内容。MSTP系统既可以加快多种线路的协同运行速度, 又可以发 挥PDH基础业务的接口优势。由此可知, MSTP系统能够有效满足用户的数据 整合需求。

WDM系统的应用价值体现在提高光纤带宽与运行频率方面。在实际应 用过程中, 既可以在光层上复用, 又可以依靠发射机对不同波长、不同频率 的信号进行光纤附着传输, 确保光纤传输质量。

2.1.3 SDH系统的特点

SDH系统是建立在SONET上的新型技术系统。SDH系统属于光纤传输数 字化传输网络。目前, 对于SDH系统的光路接口与帧结构数字传输速率有明 确的划分标准, 如此则能够增强整个网管的互通性与流畅性。

$\mathrm{SDH}$ 系统的核心原理是对信号作帧结构固定处理, 之后合理设置电路 层的复用传输速率。当光纤进入ADM系统时, 对应信号会转化为电信号, 经 过数字配线架接入用户端口, 形成完整且可靠的通信传输体系。

2. 2 传输技术的应用思路

传输技术主要应用在长途网络传输、短途网络传输和骨干线网络传输 个方面用电需要。

\section{4 结束语}

综上所述, 低压配电系统是民用高层房建电气工程的重要组成部分, 由于民用高层房建楼层多, 使其所需的电气设备更加复杂且种类繁多, 在 这种情况下, 如果不能使电气系统稳定、可靠的运行, 可能会引起安全问 题。因此需要保障低压配电设计的安全, 从而满足民用高层房建用电需求。

\section{[参考文献]}

[1]尹吴.高层建筑电气中的低压配电设计分析[J].科技风,2019,(21):187.

[2]赵卫东.当前高层建筑低压配电设计中存在的问题及不足[J].低压 配电, 2017,(01):12.

[3]刘通.浅谈高层建筑电气中的低压配电设计分析 [J]. 四川水 泥,2017,(6):99.

[4]程贝贝.低压供配电系统在高层建筑电气设计中的可靠性分析[J]. 建材与装饰,2019,(29):90-91. 
三方面。具体内容如下所述。

2.2.1短途网络传输

短途网络传输应用范围小, 容量集中, 适用于县市级中心范围。短途网 络运输的主要途径是管道光缆。管道光缆传输的缺点是传输设备升级难度 大, 传输技术不稳定。为此, 有效解决短途网络传输中传输技术的应用问题 至关重要。

\subsection{2长途网络传输}

与短途网络传输相比, 长途网络传输具有应用范围广、容量大等基 本特征。而这也对传输设备与传输技术提出了更高的标准要求。为此, 有必要加强信息通信工程建设, 改进无线网络传输效率, 提高长途网络 传输质量。

\subsection{3骨干线网络传输}

与短途网络传输和长途网络传输相比, 骨干线网络传输在诸多方面体 现出其优势。骨干线网络传输应用范围介于短途网络传输和长途网络传输 之间。相较于短途网络传输, 骨干线网络传输的设备管理更加完善与成熟, 传输设备升级较快, 且传输技术更快捷、更稳定。相较于长途网络传输, 基于骨干线网络传输集中应用于经济特区或经济发展水平较高的区域, 性 价比也相对较高。当然, 骨干线网络传输也存在一定的问题, 其中最突出的 问题就是光纤资源利用率偏低。在科技时代背景下, 要增大光纤资源利用 率, 必须在SDH网络中建设多个ASON系统, 发挥ASON系统的优势效能。

\section{3 预测传输技术的发展趋势}

3. 1 传输功能多样化

随着传输技术的深化发展, 各种新兴科技相互融合、相互促进, 且传输 功能也日趋完善与成熟。传输功能的作用体现在如下几方面: 设置增值业 务、优化资源配置、压缩投资成本、增大利用率等。

传输功能多样化与增值业务类型多样化, 极大的推动了增值业务的发 展。基于传输功能的多样化, 在不同网络系统中, 可以从多种方案中篮选匹 配度最高的方案。例如, 短距离传输网络使用与短距离传输技术相匹配; 小范围网络与小规格电缆相匹配等。传输功能多样化在资源配置方面也发 挥着重要的作用。基于传输功能的多样化特征, 采取不同的策略, 提高资源 利用率, 同时节约时间成本与投资成本。

3.2 传输产品体量微型化

传统的传输设备的体量都较大, 在运输与搭建过程中, 需要消耗更多 的人力、物力与资金, 而且数据传输难以实现一对一的信号对接。随着传
输设备的改造与传输技术的升级, 信息通信工程中的传输设备的体量越来 越微型化, 便于信号的点对点传导, 且接口转换较为便捷。部分优质的传输 产品甚至可以实现单纤芯传递。

从传输产品加工生产层面来说, 传输产品体量的微型化, 可以减少各 类材料的损耗, 降低运输费用与人工费用, 压缩生产成本, 提高性价比。总 而言之, 传输产品体量微型化, 是科技创新发展的必然结果。

3. 3 一体机传输设备

传统的传输模式主要依靠人工干预, 工作任务量繁重, 工作效率低, 投 入时间与资金较多。随着现代科技的进步,一体化传输设备越来越完善化与 成熟化, 这极大的降低了工作强度, 提高了工作效率, 压缩了投资成本。在实 践应用过程中, 单板机的种类较为多样化, 且运行速率存在较大差异。一体 化传输设备能够实现各种独立设备的整合, 形成一个完整且可靠的体系。在 这个体系中, 存在一个健全的标准界限, 可以有效控制各类设备的运作, 有 效发挥设备的监督管理作用。

一体化传输设备的工作能力较强, 在实际应用过程中, 可以对不同种 类、不同数量的资源进行分类、监管与调配。使用一体化传输设备的客户， 可以通过在线操作实现远程调控, 达到完善设备、升级技术的目的。在此 期间, 无需工作人员进入现场, 可以在很大程度上提高工作效率, 节省维修 时间, 压缩投资成本。总之, 一体化传输设备在信息通信工程中发挥着极为 关键的作用。

\section{4 结束语}

综上所述, 本文围绕传输技术在信息通信工程中的实践应用展开系统 探究。如果是固定的图像类数据的传输, 则推荐使用自动交换光网络技术 产品; 如果是移动的视频类数据的传输, 推荐使用专业的移动网络产品。 由此可知, 将传输技术拓展应用到信息通信工程中, 能够有效改善通信工 程质量。

[参考文献]

[1]罗杏通.传输技术在信息通信工程中的应用意义探讨[J].中国新通 信,2019,21(18):20.

[2]钟广鸿,通信工程中有线传输技术的应用及改进[J].计算机产品与 流通,2019,(09):37.

[3] 沈晗.传输技术在信息通信工程中的应用 [J]. 中国新通 信,2019,21(16):91. 